\section{Promoting quality blood services in the Region of the Americas}

\author{
Mirta Roses Periago ${ }^{1}$
}

1 Dr. Roses Periago became Director of the Pan American Sanitary Bureau on 1 February 2003.
Over the past several years, great progress has been made in the safety of blood for transfusion available in the Region of the Americas. The screening of blood units for infectious markers has increased. Of the 5.9 million units of blood collected in Latin America and the Caribbean in 1997, 68166 (1.1\%) were not screened for human immunodeficiency virus (HIV); 77095 (1.3\%) for hepatitis B, and 343858 (5.8\%) for hepatitis C. The corresponding figures for 2001 are 6338 $(0.09 \%)$ for HIV, $8797(0.13 \%)$ for hepatitis B, and $60112(0.88 \%)$ for hepatitis C, despite the fact that the number of units collected that year had increased to 6.8 million. The units not screened for HIV came from five countries, two of which test over $99 \%$ of the blood collected and another two at least $85 \%$. Sixteen countries do not have universal screening for hepatitis C. Of these, five do not test for hepatitis $C$ at all.

The quality of screening also appears to have improved in the Region. Training activities, development of standard operating procedures, implementation of quality standards, and the use of appropriate testing kits contribute to better laboratory performance. Nevertheless, there are still many challenges ahead in terms of achieving desired levels of safety and availability of blood.

One important issue is blood donation. Despite the fact that there is more blood available now than four years ago, there is simply not enough blood in the Region, although a few countries do collect enough to cover their needs. In general, the collection rate for Latin American and Caribbean countries is around 14 units of blood per 1000 inhabitants, well below the 50 recommended internationally. Furthermore, the majority of blood comes from persons who are asked to replace the blood intended for relatives and friends. This results in a higher risk of transmitting infections through transfusions, as well as in a higher percentage of discarded blood units. The promotion of voluntary blood donation in the countries requires a strong public education and communication component and a donor-friendly approach that includes extramural blood drives, extended operating hours, and trained personnel. In order to reach the goal of $50 \%$ voluntary blood donation, there is a need for a nationally coordinated effort, directed by 
the Ministry of Health, with support from the Ministry of Education, the Ministry of Labor, and civil society. The strategy should be geared toward taking blood donation out of the hospital environment.

"Both human and material resources must be allocated to blood services for these to have sufficient quantities of safe blood."

A second important issue relates to resources at the national level. Promotion of voluntary blood donation has a cost. Quality systems have a cost. Evaluating the impact of blood transfusion has a cost. Both human and material resources must be allocated to blood services for these to have sufficient quantities of safe blood. Currently, most countries have blood services that operate with poor efficiency by desired standards, and countries with the lowest gross national products have the most inefficient services. The economic burden imposed by the current organizational system must be carefully examined in order to realign resources and have blood banks implement a full range of services.
Reducing the number of blood processing centers is a viable alternative.

The third relevant issue is the use of blood. It is paradoxical that in the Region, where blood for transfusion is scarce, a high proportion of transfusions are given to patients who do not need them. Additionally, there is no structured system to evaluate if the blood services do contribute to patient survival, recovery, and overall well-being. Transfusion services must implement mechanisms to induce appropriate clinical use of blood, its components, and its substitutes.

"The economic burden imposed by the current organizational system must be carefully examined in order to realign resources and have blood banks implement a full range of services."

Lastly, the biggest challenge facing countries of the Region is attaining wellorchestrated, cooperative, and mutually supportive national blood programs. This calls for the ministries of health to assume a leading role in guiding all stakeholders and in bringing together the resources that are needed to manage the blood for transfusion as a valuable national resource. 